\title{
Recherche systématique des Levures dans le liquide de broncho-aspiration au cours de Pneumopathies chroniques
}

\author{
par R. MANDOUL, G. LACOSTE, M. LAHOURCADE et J.-P. MOREAU
}

L'opinion prévaut, à l'heure actuelle, que les mycoses broncho-pulmonaires, comme toutes les autres d'ailleurs, présentent un regain d'intérêt médical depuis l'emploi en thérapeutique des antibiotiques d'origine fongique, car leur nombre et leur gravité sont allés croissants.

Notre propos, en ce qui concerne les candidoses de l'appareil respiratoire, est de soumettre cette appréciation à la confrontation de quelques données précises.

Tout d'abord ces mycoses sont-elles véritablement connues depuis le début du siècle comme on l'admet d'habitude ?

Sous le nom de broncho-oïdiose, Castellani (1) a décrit en 1910 un syndrome pulmonaire tropical dont l'agent étiologique supposé fut dénommé par lui Ö̈dium tropicale; le même auteur avait déjà signalé auparavant à Ceylan la toux des dégustateurs de thé ; à cette occasion il avait isolé dans la poussière de thé divers Champignons appartenant aux genres Monilia, Aspergillus, Penicillium, Ö̈dium. Ce syndrome est pour Castellani (2) une broncho-moniliase probable (1928). Par la suite des pneumopathies aiguës désignées sous le terme de broncho-mycoses, survenant après la manipulation de céréales ou de fourrages moisis furent attribuées à l'action pathogène des Candida par Törnell (15) chez des «batteurs en grange» et par Fawcitt chez des faneurs. Avec le recul du temps, il ne fait pas de doute que les appellations anciennes d'Oïdium et de Monilia attribuées aux Candida ont favorisé et entretenu une confusion; il s'agit en réalité de Champignons Ascomycètes saprophytes, vivant dans le milieu extérieur sur les végétaux, les fruits et la terre humide. On peut rapprocher les manifestations mentionnées ci-dessus des accidents pulmonaires de la « Maladie de la canne de Provence », provoqués par le contact et l'inhalation de quantité massive de spores des innombrables colonies mycéliennes qui se développent parfois sur les roseaux stockés.

Les levures anascosporées du genre Candida (créé par Berkhout en 1923 et officialisé en 1939) comprennent moins d'une dizaine d'espèces éventuellement nuisibles pour l'homme, mais Candida albicans est de loin par sa fréquence et son aptitude pathogène la plus importante (75\% environ des levures isolées chez l'homme). Or, 
il s'agit d'un commensal très banal des muqueuses et singulièrement de l'oro-pharynx. On le rencontre aussi chez les Oiseaux (dindons, volailles) et les Mammifères sauvages ou domestiques, tel le Porc ; mais il n'est pratiquement jamais isolé du milieu extérieur à l'exception parfois de fromages ou de fruits ; les recherches déjà anciennes de Nilsby et Norden (1949) et celles plus récentes d'Ajello (1956) en témoignent. Quant aux autres levures de ce groupe, si certaines d'entre elles peuvent vivre en saprophytes dans la nature, elles n'y pullulent sûrement pas à la manière des Penicillium par exemple.

Aussi bien, la contamination humaine d'origine exogène apparaît-elle rare ou même douteuse.

Les Candidoses peuvent être considérées dans leur ensemble comme des mycoses provoquées par l'exaltation de virulence de germes jusqu'alors commensaux des cavités naturelles et de l'intestin *. Nous savons que les causes de cette exaltation sont multiples qui toutes affaiblissent les défenses de l'organisme. De là, le rôle primordial du terrain reconnu par tous les auteurs. Envisagées sous ce jour, ces mycoses apparaissent comme des affections secondes se greffant sur des états pathologiques antérieurs ou des déficiences organiques d'étiologies très diverses.

Chez l'individu sain, les levures ne dépassent guère la trachée et les grosses bronches comme l'ont fait remarquer Gâté et Coudert ; leur extension de proche en proche vers le bas est en effet continuellement limitée par l'activité des cils vibratiles et l'ascension du mucus qui rendent précaire leur présence et impossible leur installation. En fait, les ramifications bronchiques terminales et le parenchyme sont stériles; tel est l'état physiologique.

Envisageant l'état pathologique, nous avons cherché à nous renseigner sur la fréquence des Candida chez des sujets atteints de pneumopathies chroniques diverses.

Dans ce but des prélèvements systématiques ont été effectués sur 70 malades; 36 d'entre eux étaient cancéreux : 28 étaient porteurs d'épithélioma bronchique primitif, 3 d'épithélioma secondaire, 2 d'épithélioma œsophagien avec envahissement trachéal, 1 d'un lymphosarcome, 2 d'épithélioma laryngé. Aucun ne présentait de candidose clinique, aucun n'était cachectique; la plupart étaient en cours de cure soit télécobalthérapique, soit chimiothérapique, soit radiothérapique.

Chez les autres patients on relevait : 13 bronchorrhées mucopurulentes avec emphysème, 2 bronchectasies surinfectées, 4 pneumonies chroniques en foyers, 2 abcès du poumon, 1 tuberculose pulmonaire, 3 états asthmatiques. Dans ce deuxième groupe tous avaient subi soit per os, soit par voie parentérale, une antibiothérapie associée souvent aux corticoïdes, d'une durée de 1 à 4 mois : tétracyclines, pénicilline, streptomycine, PAS, INH, sulfamides selon les cas.

(*) Exception doit être faite, pour quelques petites épidémies infantiles dans les crèches, et dans quelques cas, de contamination directe d'infirmières, ou de mères par leur nourrisson, ou bien encore lors de rapports sexuels. 


\section{Prélèvement.}

Il a été effectué dans les bronches souches ou dans les bronches lobaires. Pour éviter toute souillure du bronchoscope au passage, un tube stérile de matière plastique était introduit dans la gaine métallique d'un aspirateur de Negus, sa partie distale demeurant en léger retrait de l'extrémité de la gaine et par conséquent protégée par elle. Après la mise en place, l'aspirateur était poussé hors de sa gaine pour y être ramené dès le prélèvement opéré. Les sécrétions ainsi recueillies étaient ensuite refoulées par un jet de sérum physiologique dans un flacon stérile. Nous avons d'autre part vérifié que la stérilisation de notre appareillage par le formol était bien satisfaisante puisque nul microorganisme ne se développait à partir d'une eau stérile de lavage ; 78 prélèvements ont été réalisés, certains sujets ayant fait l'objet de deux prospections successives.

Un prélèvement buccal était effectué au préalable sur chaque patient et mis en culture conjointement. Cette recherche comparative des levures buccales et bronchiques chez un même individu ne semble pas avoir retenu l'attention des auteurs. Le plus souvent les expectorations sont recueillies directement ou bien après lavage de la bouche par une solution antiseptique. A notre connaissance, seul Mankowski (9), qui nous a précédé dans cette investigation systématique, a opéré par voie endoscopique, sans toutefois signaler que des précautions spéciales aient été prises pour éviter la souillure du bronchoscope.

\section{Isolement et identification des souches.}

Nous avons suivi le processus classique: étalement de chaque prélèvement en boîte de Pétri sur gélose de Sabouraud sucrée à $2 \%$ (glucose et maltose en parties égales); repiquage des colonies de type levure, après purification en cas de nécessité, sur le milieu de Gorodkova pour la mise en évidence des espèces ascosporées, et sur le milieu P.C.B. pour solliciter la production d'un pseudo-mycélium caractéristique du genre Candida et révéler éventuellement l'élaboration des chlamydospores du groupe Candida albicans. Lorsque l'identification morphologique n'était pas possible, chaque souche était soumise au zymogramme ; dans quelques cas, un auxanogramme des sucres fut nécessaire pour assurer le diagnostic spécifique.

\section{Résultats.}

En définitive, nous avons isolé et identifié 62 souches à partir des prélèvements buccaux et 26 souches dans les produits de broncho-aspiration.

Le tableau ci-dessous en indique le détail.

Si nous comparons maintenant ces résultats à ceux de Mankowski, nous constatons leur quasi similitude; la fréquence des levures dans les bronches de sujets atteints de pneumopathies chroniques semble se situer un peu en dessous de $20 \%$ des cas; il faut noter encore que la plupart du temps il s'agit d'une présence discrète et non d'une culture massive. 


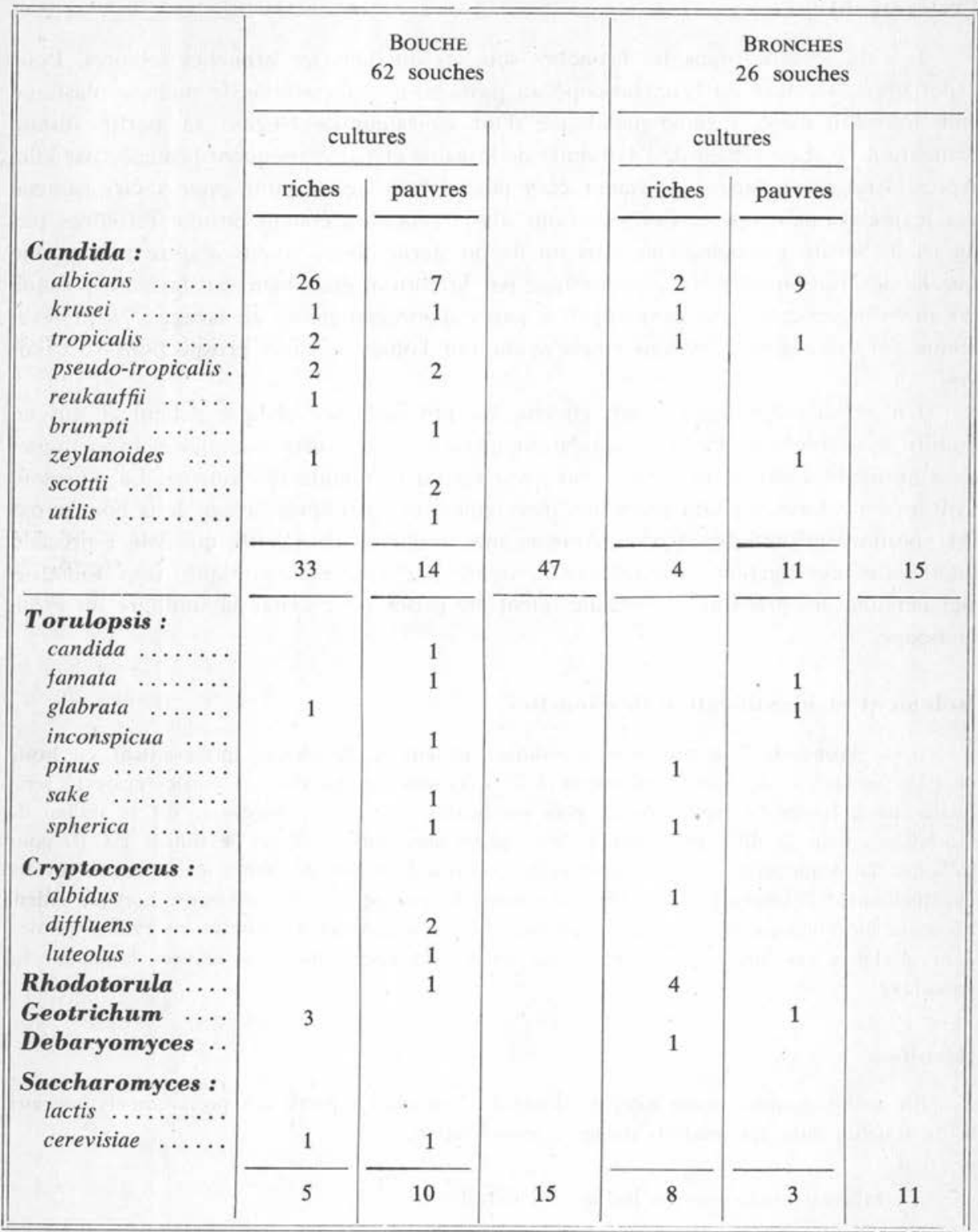

Mais la bronchoscopie ne pouvant prétendre explorer au-delà de l'orifice des bronches segmentaires, il faut s'adresser à d'autres moyens pour compléter nos connaissances sur ce point ; c'est ce qu'ont réalisé d'une part Monod et ses collaborateurs (2), 
d'autre part Marmet et Rioux (10) en ensemençant des broyats de pièces d'exérèse pulmonaire ; les premiers ont obtenu 3 cultures de Candida sur 62 pièces et les seconds une seule sur 80 .

\section{Conclusions.}

De l'ensemble des données précédentes, nous nous croyons autorisés à tirer les considérations suivantes :

Les levures du genre Candida et singulièrement Candida albicans peuvent être abondantes dans la bouche et rares ou même absentes dans les bronches; chez un même sujet, on peut rencontrer dans la bouche et dans les bronches soit les mêmes espèces, soit des espèces différentes. Il s'ensuit que seul le prélèvement endoscopique peut fournir des renseignements précis, car celui des crachats expectorés risque de susciter un diagnostic erroné.

$\mathrm{Au}$ niveau de l'arbre trachéo-bronchique pratiquement stérile, le rôle favorisant éventuel des antibiotiques fongiques dans l'éclosion des Candidoses broncho-pulmonaires ne peut être envisagé sous l'angle d'une abolition de la concurrence vitale des microorganismes comme dans les «Moniliases intestinales ou cavitaires. Hormis le cas de la voie hématogène heureusement fort rare, il est probable qu'une lésion antérieure si minime soit-elle et quelle que soit son étiologie peut seule permettre le développement d'une culture in vivo * le pouvoir pathogène des Candida peut s'exprimer alors d'autant plus aisément que les défenses générales de l'organisme sont plus déficientes. Mais l'expérience montre que même au cours des diverses pneumopathies chroniques, tuberculose comprise, ces conditions locales et générales se trouvent rarement réunies.

Aussi bien peut-on penser que les candidoses bronchiques et pulmonaires demeureront à l'avenir ce qu'elles ont toujours été, c'est-à-dire des mycoses d'exception.

\section{Bibliographie}

1. Castellani A., 1910. - Observations on Tropical Broncho-oïdiosis. British med. J., 2, 868.

2. - 1928. - Fungi and fungous diseases. Arch. Dermat. and Syph., 17, 79.

3. Coudert J., Despierres G., Saes H., Hollard J., 1957. - Recherches sur la flore levuriforme bronchique en milieu sanatorial. Sem. Hôp. Paris, 49, 2978.

4. D'Eshougues J. R., Zaffran A. et Cohen-Adad, 1951. - Blastomycose pulmonaire à C. tropicalis. Bull. et Mém. Soc. Méd. Hôp. Paris, 67, 1128.

5. Drouet E., 1957. - Biologie des infections à Candida. II. Sur les manifestations pathologiques et les conditions étiologiques et pathogéniques de 175 cas de Candidose. Sem. Hôp., 20, 807.

${ }^{*}$ ) Nous avons précédemment soutenu la même thèse vis-à-vis de l'Aspergillose bronchique. 
6. Gaté J. et Coudert J., 1958. - Traitement des Mycoses. G. Doin Edit., Paris, p. 149.

7. Graciansky (de) P. et Delaporte J., 1956. - Accidents à levures des traitements par les antibiotiques. Masson Edit.

8. Herbeuval R., Herbeuval H., Cuny G., Debry G. et Manciaux M., 1955. - Pneumopathie aiguë à C. pseudotropicalis. J. Fr. Méd. Thor., 9, 160.

9. Mankowski Z.-T., 1953. - La flore fongique des bronches. Sem. Hôp. Paris, 29, 4, 186.

10. Marmet A., Plane J., Rioux J.-A., 1956. - L'infection tuberculeuse et l'antibiothérapie pré-opératoire ne favorisent pas le développement des Candida au sein du parenchyme pulmonaire. Montpellier Méd., 49, 503.

11. Monod O., Pesle G. D., Presse M., 1954. - Contribution à l'étude de la flore microbienne et mycologique dans les pièces d'exérèse en chirurgie pulmonaire. La Presse Méd., 62, 77, 1591.

12. ORIE N. G. M., 1952. - Candida (Monilia). Infection of Respiratory tract. Diseases of the Chest., 22, 107.

13. Raton D., 1951. - Maladie des «batteurs en grange». J. Méd. et Chir. thoraciques, 5, 430.

14. Rioux J. A., Verdier P., Plane J., 1954. - Sur la fréquence des infestations saprophytiques à Candida chez les tuberculeux pulmonaires. Le Poumon, 10, 101.

15. Törnell E., 1946. - Thresher's lung. Acta medica Scandinavia, 125, 3, 191.

16. Woods J. W., Manning I. H. and Patterson C. N., 1957. - Monilial infections complicating the therapeutic use of antibiotics. J.A.M.A., 145, 207.

(Fondation Bergonié et Laboratoire de Parasitologie de la Faculté mixte de Médecine et de Pharmacie de Bordeaux) 